\title{
Calagem e adubação no crescimento de espinheira-santa [Maytenus ilicifolia (Schrad.) Planch.] em casa de vegetação
}

\author{
BENEDETTI, E.L. ${ }^{1 *}$; SERRAT, B.M. ${ }^{2}$; SANTIN, D. ${ }^{1}$; BRONDANI, GE. ${ }^{3}$; REISSMANN, C.B. ${ }^{2}$; BIASI, L.A. ${ }^{4}$ \\ ${ }^{1}$ Universidade Federal de Viçosa (UFV), Departamento de Solos e Nutrição de Plantas, Avenida Peter Henry Rolfs, \\ s/n Campus Universitário, Viçosa/MG, 36570-000, elibettiagro@yahoo.com.br *(Bolsista Capes). ${ }^{2}$ Universidade \\ Federal do Paraná (UFPR), Departamento de Solos e Engenharia Agrícola. ${ }^{3}$ Esalq (USP), Departamento de Ciências \\ Florestais. ${ }^{4}$ Universidade Federal do Paraná (UFPR), Departamento de Fitotecnia.
}

\begin{abstract}
RESUMO: Devido a pouca informação existente sobre adubação para espinheira-santa, e a crescente procura desta espécie, o objetivo deste trabalho foi avaliar o efeito da calagem, adubação orgânica, mineral e omissão de nutrientes sobre o crescimento de Maytenus ilicifolia. As mudas foram produzidas por sementes e, em agosto de 2006, foram transferidas para vasos com $8 \mathrm{dm}^{3}$ contendo Latossolo Vermelho Distrófico. O experimento foi conduzido em casa de vegetação no delineamento inteiramente casualizado em arranjo bifatorial, totalizando 12 tratamentos com 4 repetições, cujos tratamentos foram: sem adubação com calagem ( $T 1$ ), sem adubação e sem calagem (T7), adubação orgânica (T2 e T8), adubação mineral NPK (T3 e T9), mineral -N (T4 e $\mathrm{T} 10$ ), mineral $-\mathrm{P}$ (T5 e T11) e mineral -K (T6 e T12). Os tratamentos $\mathrm{T} 1$ ao T6 receberam calcário na dose equivalente a 7,06 tha-1 de carbonato de cálcio e os tratamentos T7 ao T12 foram testados na ausência de calagem. Após 314 dias da instalação do experimento, foram analisadas as variáveis: massa seca foliar (MSF), massa seca do caule (MSC) e da raiz (MSR), massa seca da parte aérea (MSPA), área foliar (AF), volume radicular $(\mathrm{VR})$, altura $(H)$, diâmetro $(\mathrm{D})$, relação raiz parte aérea $(\mathrm{R} / \mathrm{PA})$ e relação altura diâmetro $(\mathrm{H} / \mathrm{D})$. A partir dos resultados conclui-se que a calagem, quando utilizada como prática isolada, não resulta em benefícios significativos ao crescimento das plantas. Na presença de calagem, tanto a adubação mineral quanto a orgânica podem ser utilizadas. Na ausência de calagem, a adubação orgânica mostrou-se mais favorável em estimular o crescimento das plantas. Em condições de adubação mineral, independente da presença ou não da calagem, o fósforo mostrou-se importante à nutrição de espinheira-santa.
\end{abstract}

Palavras-chave: plantas medicinais, fertilização mineral, fertilização orgânica

\begin{abstract}
Liming and fertilization on "espinheira-santa" [Maytenus ilicifolia (Schrad.) Planch.] growth in greenhouse. Since there is scarce information about fertilization for "espinheira-santa" and an increasing search for this species, the present study aimed to evaluate the effect of liming, organic and mineral fertilization, and nutrient omission on Maytenus ilicifolia growth. Seedlings were produced from seeds, and in August 2006 they were transferred to $8 \mathrm{dm}^{3}$ pots containing Dystrophic Red Latosol. The experiment was carried out in a greenhouse and the design was completely randomized, in bi-factorial arrangement, totaling 12 treatments and four replicates; treatments were: without fertilization and with liming (T1), without fertilization and without liming (T7), organic fertilization (T2 and T8), mineral fertilization NPK (T3 and T9), mineral $-\mathrm{N}$ (T4 and T10), mineral - $\mathrm{P}$ (T5 and T11) and mineral - $\mathrm{K}$ (T6 and T12). T1 to T6 received lime at the dose equivalent to $7.06 \mathrm{tha}^{-1}$ calcium carbonate, and T7 to T12 were tested in the absence of liming. After 314 days of the experiment installation, the following variables were analyzed: leaf dry mass (LDM), stem (STDM) and root dry mass (RDM), shoot dry mass (SDM), leaf area (LA), root volume (RV), height $(H)$, diameter $(D)$, root and shoot ratio $(R / S)$, and height and diameter ratio $(H / D)$. Liming alone does not result in significant benefits to plant growth. In the presence of liming, both mineral and organic fertilization can be employed. In the absence of liming, organic fertilization showed to be more favorable in stimulating plant growth. Under mineral fertilization conditions, irrespective of the presence or absence of liming, phosphorus showed to be important for the nutrition of "espinheira-santa".
\end{abstract}

Key words: medicinal plants, mineral fertilization, organic fertilization

Recebido para publicação em 08/05/2008

Aceito para publicação em 16/01/2009

Rev. Bras. PI. Med., Botucatu, v.11, n.3, p.269-276, 2009. 


\section{INTRODUÇÃO}

Maytenus ilicifolia (espinheira-santa) caracteriza-se por ser subarbusto ou árvore ramificada desde a base. É espécie nativa da América do Sul, de ocorrência no Sul do Brasil, além do Paraguai, Uruguaie leste da Argentina (Carvalho-Okano, 1992). De acordo com Radomski (2006), esta espécie apresenta significativa plasticidade ambiental, sendo que ocorre em solos com distintas condições de fertilidade.

O Paraná destaca-se como o maior coletor de espinheira-santa do Brasil (Corrêa Júnior et al., 1991), sendo esta, a quarta espécie medicinal nativa mais comercializada no país (Steenbock \& Reis, 2004).

A confirmação das propriedades terapêuticas das folhas de espinheira-santa na proteção e tratamento de úlceras gástricas foi relatada por Carlini (1988). Esta confirmação gerou uma grande demanda por produtos à base de espinheira-santa (Scheffer, 2004) o que vem aumentando a pressão sobre as populações naturais, visto que a maior parte do produto é oriunda do extrativismo (Steenbock \& Reis, 2004), prática essa que pode levar a espécie à extinção.

Desta forma, seu cultivo é a maneira mais adequada de atender a demanda do mercado (Montanari Júnior, 1999). Para isto, um dos primeiros passos é o acompanhamento do crescimento, sendo a influência da fertilização nessa fase, um importante aspecto técnico a ser investigado.

Embora testes com adubações em $M$. aquifolia tenham resultado em efeitos positivos ao crescimento (Menezes et al., 1992), ainda não existe recomendação de adubação para nenhuma espécie do gênero Maytenus.

Algumas práticas de manejo, que visam adequar o ambiente às necessidades das plantas podem ser utilizadas. Acalagem é uma delas, tendo seus efeitos já conhecidos (Tedesco \& Bissani, 2004; Sousa et al., 2007), principalmente para culturas anuais. Alguns estudos com várias espécies florestais demonstraram resultados diversos, com respostas positivas (Furtini Neto et al., 1999; Cruz et al., 2004) e negativas (Reissmann et al., 1997) à aplicação de calagem.

A adubação orgânica possui várias vantagens, como o aproveitamento do valor fertilizante, propiciando a reciclagem de nutrientes no sistema produtivo, evitando a contaminação ambiental (Selbach \& Sá, 2004), além de diminuir os custos de produção, visto ser encontrado na maioria das propriedades. Respostas positivas à utilização de substratos a base de compostos orgânicos foram obtidos por Santin et al. (2004) em erva-mate.

Apesar de a adubação química ser uma prática bastante difundida no setor agrícola, segundo Blank et al. (2006) no Brasil há pouca informação a respeito para plantas medicinais.
Sendo assim, o objetivo deste estudo foi avaliar o efeito da presença e ausência de calagem combinadas com adubação orgânica, adubação mineral e omissão de N, P e Kem Latossolo Vermelho Distrófico, no crescimento da parte aérea e das raízes de plantas jovens de M. ilicifolia.

\section{MATERIAL E MÉTODO}

\section{Dados gerais}

O experimento foi realizado em casa-devegetação na Universidade Federal do Paraná, em Curitiba-PR, no período de 25 de agosto de 2006 a 05 de julho de 2007. As mudas de M. ilicifolia (Schrad.) Planch. (espinheira-santa) foram produzidas por sementes, as quais foram coletadas de matrizes localizadas em vários locais do município de São Mateus do Sul-PR. No início de fevereiro de 2006, as mesmas foram semeadas em sacos plásticos $(12 \mathrm{x}$ $8 \mathrm{~cm}$ ), com substrato comercial para espécies nativas. Permaneceram neste substrato por aproximadamente 190 dias. Esta etapa foi realizada no viveiro da empresa Baldo S. A., localizado em São Mateus do Sul-PR.

O substrato utilizado para condução do experimento foi Latossolo Vermelho Distrófico, analisado de acordo com Embrapa (1997) com as seguintes características: $\mathrm{pH}_{\text {em }} \mathrm{CaCl}_{2}=4,00 ; \mathrm{Al}^{+3}$, $\mathrm{H}^{+}+\mathrm{Al}^{+3}, \mathrm{Ca}^{+2}, \mathrm{Mg}$, Ke T respectivamente de 3,9; 12,1; 0,$3 ; 0,1 ; 0,12$ e $12,6 \mathrm{cmol}_{\mathrm{c}} \mathrm{dm}^{-3} ; \mathrm{P}=1,9 \mathrm{mg} \mathrm{dm}^{-3} ; \mathrm{C}=$ $35,3 \mathrm{~g} \mathrm{dm}^{-3}$; saturação por bases $=4,0 \%$; areia e argila, respectivamente, de 52,5 e $825,0 \mathrm{~g} \mathrm{~kg}^{-1}$.

O experimento foi conduzido no delineamento inteiramente casualizado (Storck et al., 2000) no arranjo bifatorial (6x2) com 4 repetições, sendo os fatores constituídos por composições (tipos) de adubações e aplicação de calagem, totalizando 12 tratamentos.

Os solos dos tratamentos $\mathrm{T} 1$ ao $\mathrm{T} 6$ receberam $27,1 \mathrm{~g} \mathrm{vaso}^{-1}$ de calcário dolomítico (PRNT = 104\%) resultante do cálculo para elevar a saturação por bases do solo a 60\% (Comissão de Química e Fertilidade do Solo, 2004 ). Os solos dos tratamentos T7 ao T12 permaneceram sem calagem.

Quanto aos tipos de adubação, os tratamentos foram compostos por: sem adubação (T1 e T7), adubação orgânica (T2 e T8), mineral NPK (T3 e T9), mineral -N (T4 e T10), mineral -P (T5 e T11) e mineral-K (T6 e T12). Os tratamentos T1 ao T6 foram testados na presença de calagem e de T7 ao T12 na ausência de calagem, conforme representado na Tabela 1.

Para compor os tratamentos com adubação mineral (Tabela 1), baseou-se na recomendação da (Comissão de Química e Fertilidade do Solo, 2004 ) para o plantio de erva-mate, tendo em vista que ambas 
TABELA1. Descrição dos tratamentos aplicados ao solo para o cultivo de mudas de Maytenus ilicifolia, Curitiba, Paraná.

\begin{tabular}{|c|c|c|c|c|c|c|c|c|}
\hline \multirow{4}{*}{ Adubações } & \multicolumn{8}{|c|}{ Calagem } \\
\hline & \multicolumn{4}{|c|}{ Presença* } & \multicolumn{4}{|c|}{ Ausência } \\
\hline & \multirow{2}{*}{ Tratamento } & $\mathrm{N}$ & $\mathrm{P}_{2} \mathrm{O}_{5}$ & $\mathrm{~K}_{2} \mathrm{O}$ & \multirow{2}{*}{ Tratamento } & $\mathrm{N}$ & $\mathrm{P}_{2} \mathrm{O}_{5}$ & $\mathrm{~K}_{2} \mathrm{O}$ \\
\hline & & $\ldots .$. & $\mathrm{kg} \mathrm{ha}$ & $\ldots \ldots$ & & \multicolumn{3}{|c|}{.. $\mathrm{kg} \mathrm{ha}{ }^{-1}$} \\
\hline Ausência & $\mathrm{T} 1$ & 0 & 0 & 0 & T7 & 0 & 0 & 0 \\
\hline Orgânica & $\mathrm{T} 2$ & 20 & 7 & 53 & T8 & 20 & 7 & 53 \\
\hline Mineral NPK & T3 & 20 & 30 & 20 & T9 & 20 & 30 & 20 \\
\hline Mineral -N & $\mathrm{T} 4$ & 0 & 30 & 20 & T10 & 0 & 30 & 20 \\
\hline Mineral -P & T5 & 20 & 0 & 20 & T11 & 20 & 0 & 20 \\
\hline Mineral $-K$ & T6 & 20 & 30 & 0 & $\mathrm{~T} 12$ & 20 & 30 & 0 \\
\hline
\end{tabular}

* Dose de 7,06 t ha' ${ }^{1}$.

ocorrem em Floresta Ombrófila Mista (Carvalho, 1980; Galvão et al., 1989; Oliveira \& Rotta, 1985), aliada a falta de recomendação específica para espinheirasanta. As fontes de N, P e K utilizadas para os tratamentos com adubação mineral foram uréia, superfosfato triplo e cloreto de potássio, respectivamente. Para a adubação orgânica (Tabela 1), adicionou-se $41,6 \mathrm{~g}$ vaso $^{-1}$ de esterco de ovino, 0 qual possuía 58,3; 6,6;64,7; 16,7 e $6,8 \mathrm{~g} \mathrm{~kg}^{-1}$, respectivamente, de N, P, K, Ca e Mg, e valores de 2609; 1020; 22 e $270 \mathrm{mg} \mathrm{kg}^{-1}$, respectivamente de $\mathrm{Fe}, \mathrm{Mn}, \mathrm{Cu}$ e Zn.

\section{Preparo e instalação do experimento}

O solo foi submetido à agitação mecânica utilizando peneira de $7 \mathrm{~mm}$ de espessura de malha. Após secagem ao ar, aplicou-se a dose de calcário calculada no volume de solo correspondente aos tratamentos com adição de calagem. Incubou-se o solo por 48 dias mantendo a umidade em torno de $50 \%$ da capacidade de campo, procedimento também realizado para a porção de solo que não recebeu calagem.

Os vasos, representando as repetições, foram preenchidos com $8 \mathrm{dm}^{3}$ de solo seco ao ar, homogeneizados com o adubo correspondente a cada tratamento. Transferiram-se quatro mudas para cada vaso, as quais tiveram as raízes lavadas e podadas, deixando-as com aproximadamente $10 \mathrm{~cm}$ de comprimento. Após 40 dias do transplante, retirouse de cada vaso a planta que obteve o menor crescimento. Durante a condução do experimento o solo foi mantido sempre úmido, com adição de mesmo volume de água desionizada para todos os vasos.

\section{Variáveis analisadas}

Após 314 dias da instalação mediu-se a altura total $(\mathrm{H})$ com régua graduada $(\mathrm{cm})$ da superfície do solo até o ápice da planta. Já o diâmetro (D) do colo foi medido a $0,5 \mathrm{~cm}$ do solo com a utilização de paquímetro $(\mathrm{mm})$, determinando-se a relação altura pelo diâmetro $(H / D)$, conforme sugerido por Carneiro (1995).

Nesta mesma ocasião coletaram-se as folhas e separou-se o caule da raiz. Para retirada das raízes, o solo foi umedecido e, posteriormente, passado em peneira de $2 \mathrm{~mm}$ para retenção das raízes, as quais foram lavadas em água corrente. Obteve-se o volume radicular (VR) pelo volume de água deslocada com a imersão das raízes em uma proveta de $250 \mathrm{~mL}$. O restante do material foi lavado com água desionizada.

A área foliar (AF) foi medida no aparelho Winrhizo. Posteriormente, levou-se o material para estufa com ventilação forçada a temperatura de $60^{\circ} \mathrm{C}$, onde permaneceu até massa constante, momento em que se determinou a massa seca foliar (MSF), massa seca do caule (MSC) e massa seca radicular (MSR). Com a soma da MSF e MSC obteve-se a massa seca da parte aérea (MSPA) possibilitando determinar a relação raiz parte aérea (R/PA).

Os dados foram submetidos à análise de variância (ANOVA), tendo as médias sido discriminadas pelo teste de agrupamento de Duncan, a $5 \%$ de probabilidade de erro, pelo programa estatístico SOC (Embrapa, 1990).

\section{RESULTADO E DISCUSSÃO}

Os resultados da análise da variância encontram-se na Tabela 2, onde é possível verificar que houve interação entre os tipos de adubações (AD) e o efeito da calagem (EC) para todas as características amostradas. Assim, a discussão segue abordando a influência da presença e ausência da calagem para cada tipo de adubação e, após, a influência dos tipos de adubações na presença e na ausência de calagem. 
TABELA 2. Resultados da análise de variância (ANOVA) para massa seca foliar (MSF), massa seca do caule (MSC), massa seca da raiz (MSR), massa seca da parte aérea (MSPA), relação raiz parte aérea (R/PA), área foliar $(\mathrm{AF})$, volume radicular $(\mathrm{VR})$, altura $(\mathrm{H})$, diâmetro $(\mathrm{D})$ e relação altura diâmetro $(\mathrm{H} / \mathrm{D})$ em mudas de espinheira-santa (Maytenus ilicifolia).

\begin{tabular}{|c|c|c|c|c|c|c|c|c|c|c|c|}
\hline \multirow{2}{*}{ Fv } & $G$ & $\mathrm{MSF}^{(1)}$ & MSC & MSR & MSPA & R/PA & \multirow{2}{*}{$\begin{array}{l}\mathrm{AF}^{(1)} \\
\mathrm{cm}^{2} \\
\end{array}$} & \multirow{2}{*}{$\frac{\mathrm{VR}}{\mathrm{cm}^{3}}$} & \multirow{2}{*}{$\begin{array}{c}\mathrm{H} \\
\mathrm{cm} \\
\end{array}$} & \multirow{2}{*}{$\frac{\mathrm{D}}{\mathrm{mm}}$} & \multirow{2}{*}{$\frac{\mathrm{H} / \mathrm{D}}{\mathrm{mm} \mathrm{cm}^{-1}}$} \\
\hline & & $\ldots$. & ........ & .g planta ${ }^{-1}$ & & $\ldots \ldots$. & & & & & \\
\hline$A D$ & 5 & $0,218^{*}$ & $135,87^{*}$ & $186,88^{\star}$ & $546,70^{\star}$ & $0,017^{\star}$ & $19,72^{\star}$ & $1063,38^{*}$ & $813,59^{\star}$ & $3,579^{\star}$ & $6,69^{\star}$ \\
\hline EC & 1 & $0,972^{*}$ & $519,95^{*}$ & $536,87^{\star}$ & $2325,19^{*}$ & $0,008^{*}$ & $104,99^{*}$ & $1026,75^{\star}$ & $1354,79^{*}$ & $14,192^{*}$ & $1,03^{*}$ \\
\hline$D^{*} E C$ & 5 & $0,062^{*}$ & $42,68^{*}$ & $37,98^{*}$ & $136,69^{*}$ & $0,017^{*}$ & $4,360^{*}$ & $542,5^{*}$ & $328,78^{*}$ & $1,125^{*}$ & $5,53^{*}$ \\
\hline Erro & 36 & 0,011 & 8,56 & 5,83 & 19,26 & 0,005 & 0,980 & 61,76 & 56,43 & 0,154 & 2,23 \\
\hline Média & - & 17,95 & 12,81 & 13,58 & 31,19 & 0,42 & 11,87 & 36,08 & 62,69 & 5,46 & 11,48 \\
\hline $\mathrm{CV}(\%)$ & - & 7,91 & 22,84 & 17,78 & 14,06 & 16,96 & 8,34 & 21,78 & 11,98 & 7,20 & 13,02 \\
\hline
\end{tabular}

"*" valor de F significativo ao nível de $5 \%$ de probabilidade de erro; (1) dados transformados por $\sqrt{\frac{p}{10}}$ pelo teste de Bartlett, ao nível de $5 \%$ de probabilidade. $\mathrm{FV}=$ Fator de variação, $\mathrm{GL}=$ graus de liberdade, $\mathrm{AD}=$ adubação, $\mathrm{EC}=$ efeito da calagem, $\mathrm{CV}=\mathrm{coeficiente}$ de variação, $\mathrm{p}=$ dado amostrado.

Influência da presença e ausência da calagem para cada tipo de adubação

$\mathrm{Na}$ Tabela 3 encontra-se o resultado da análise de solo realizada para cada tratamento após o cultivo. Nas Tabelas 4, 5 e 6 apresentam-se os resultados da análise estatística das variáveis de crescimento avaliadas.

Ao comparar os tratamentos sem adubação, observa-se que com calagem (T1) as plantas aumentaram a produção de AF e o D. Entretanto o VR apresentou redução, sendo que a calagem não influenciou as demais variáveis de crescimento
(Tabelas 4, 5 e 6). Esse efeito positivo da calagem permite verificar que a correção do solo pode ser adotada visando, principalmente, a obtenção de maior $\mathrm{AF}$, a qual pode aumentar o potencial fotossintético da planta, podendo vir a refletir na produção da planta adulta. Este aumento da AF e D concordam com Montanari Júnior et al. (2004) de que a espinheirasanta responde a correção do solo.

Pela avaliação do crescimento das plantas percebe-se que a adubação orgânica pode ser uma prática menos dependente da correção do solo para sua eficácia, uma vez que apenas para a MSR e R/

TABELA 3. Médias de $\mathrm{pH}, \mathrm{Al}^{+3}, \mathrm{Ca}^{+2}, \mathrm{Mg}^{+2}, \mathrm{~K}^{+}, \mathrm{P}$ e $\mathrm{C}$ do solo após aplicação dos tratamentos e cultivo de Maytenus ilicifolia, por 314 dias. Curitiba, Paraná*.

\begin{tabular}{ccccccccc}
\hline \multicolumn{2}{c}{ Tratamento } & $\mathrm{pH}$ & $\mathrm{Al}^{+3}$ & $\mathrm{Ca}^{+2}$ & $\mathrm{Mg}^{+2}$ & $\mathrm{~K}^{+}$ & $\mathrm{P}$ & $\mathrm{C}$ \\
\hline Calagem & Adubação & $\mathrm{CaCl}_{2}$ & $\ldots \ldots \ldots \ldots \ldots \ldots . . \mathrm{cmol}_{\mathrm{c}} \mathrm{dm}^{-3} \ldots \ldots \ldots \ldots \ldots$ & $\mathrm{mg} \mathrm{dm}^{-3}$ & $\mathrm{~g} \mathrm{dm}^{-3}$ \\
\hline \multirow{5}{*}{ Presença } & Ausência & 4,40 & 1,00 & 2,60 & 1,80 & 0,11 & 1,60 & 32,3 \\
& Orgânica & 4,60 & 0,80 & 3,20 & 2,00 & 0,42 & 2,90 & 32,9 \\
& Mineral NPK & 4,40 & 1,20 & 2,80 & 1,90 & 0,25 & 10,80 & 34,7 \\
& Mineral -N & 4,60 & 0,90 & 2,60 & 1,80 & 0,24 & 9,80 & 34,1 \\
& Mineral -P & 4,40 & 1,30 & 2,50 & 1,70 & 0,30 & 1,60 & 32,9 \\
& Mineral -K & 4,40 & 1,30 & 2,70 & 1,80 & 0,10 & 10,40 & 32,9 \\
\hline \multirow{4}{*}{ Ausência } & Ausência & 4,00 & 4,10 & 0,50 & 0,20 & 0,11 & 1,50 & 28,7 \\
& Orgânica & 3,90 & 3,60 & 0,70 & 0,50 & 0,46 & 3,20 & 30,5 \\
& Mineral NPK & 3,80 & 4,20 & 0,70 & 0,30 & 0,33 & 12,50 & 30,5 \\
& Mineral - $\mathrm{N}$ & 3,90 & 4,10 & 0,80 & 0,40 & 0,31 & 11,10 & 31,1 \\
& Mineral -P & 3,70 & 5,00 & 0,50 & 0,30 & 0,36 & 1,90 & 29,3 \\
& Mineral -K & 3,80 & 4,40 & 0,80 & 0,30 & 0,13 & 12,90 & 20,5 \\
\hline
\end{tabular}

* Amostras de solo composta de 4 repetições de cada tratamento. 
PA foi observado superioridade na aplicação de calagem (Tabelas 4 e 6), não diferindo para as demais variáveis. No entanto, o fato das mudas possuírem maior MSR e, conseqüentemente, maior relação $\mathrm{R} /$ PA poderá ser fator determinante para a sobrevivência no campo, o que remete a necessidade de investigações futuras para o acompanhamento do desenvolvimento das plantas após o plantio permanente.

O efeito dos adubos orgânicos, de acordo com Motta \& Serrat (2006) pode ainda estar associado à presença de outros nutrientes, além do $\mathrm{N}, \mathrm{Pe} \mathrm{K}, \mathrm{O}$ que pode ter influenciado na resposta positiva da adubação orgânica neste estudo. Além disso, Ernani \& Gianello (1983); Miyazawa et al. (1992); Franchini et al. (1999) e Mello \& Vitti, (2002) citam que a atividade do $\mathrm{Al}^{+3}$ e seu efeito tóxico diminuem com a aplicação de resíduos orgânicos ao solo, pela complexação deste, tendência essa observada no solo para os tratamentos com adubação orgânica (Tabela 3).

Para adubação mineral NPK, a prática da calagem mostrou-se benéfica à espinheira-santa para a maioria das variáveis analisadas (Tabelas 4, 5 e 6). Esse resultado pode ser indicativo de que, ao contrário da adubação orgânica, a mineral é dependente de calagem, principalmente nestas condições de solo ácido, para a melhoria da eficiência da fertilização.

A prática da calagem não neutralizou totalmente o Al e nem proporcionou elevação do $\mathrm{pH}$ superior a 4,60 (Tabela 3). Mesmo assim, essa neutralização parcial pode ter sido a responsável pelas respostas positivas à maioria das variáveis de crescimento avaliadas nas condições de calagem associada à adubação mineral. O que pode explicar tal resposta é que essa espécie tolera solos levemente ácidos (Bittencourt, 2000; Steenbock, 2003; Silva Júnior, 2003), indicando que neste tratamento o solo em questão atingiu valores de fertilidade suficientes para seu crescimento.

\section{Influência dos tipos de adubações na presença e ausência de calagem}

Percebe-se que a utilização de adubos, tanto orgânico quanto mineral, associados à correção do solo demonstrou eficiência em estimular o crescimento, visto a superioridade em relação ao tratamento que recebeu apenas calagem (T1) na maioria das variáveis analisadas (MSF, MSC, MSR, MSPA, VR, AF e R/PA) (Tabelas 4, 5 e 6).

Entretanto, as plantas submetidas à adubação mineral com calagem, apresentaram maior produção de MSC, MSPAe $\mathrm{H}$, em relação à adubação orgânica, não diferindo da mesma para as demais variáveis. Neste aspecto, a forma prontamente disponível dos nutrientes na adubação mineral (Kiehl, 1993) juntamente com a maior disponibilidade de $P$ (Tabela 1), e os benefícios da calagem (Sousa et al., 2007) podem ter sido responsáveis em proporcionar o maior valor dessas variáveis para esse período.

Ao comparar a adubação mineral completa com omissões de N, P e K, observou-se que os maiores efeitos negativos ocorreram, principalmente, na adubação com omissão de $P$, tanto na presença quanto na ausência da calagem, demonstrando que

TABELA4. Massa seca foliar (MSF), massa seca do caule (MSC), massa seca da raiz (MSR) e massa seca da parte aérea (MSPA) para mudas de Maytenus ilicifolia, cultivadas por 314 dias. Curitiba, Paraná1.

\begin{tabular}{|c|c|c|c|c|c|}
\hline \multicolumn{2}{|c|}{ Tratamentos } & MSF & MSC & MSR & MSPA \\
\hline Calagem & Adubação & \multicolumn{4}{|c|}{ 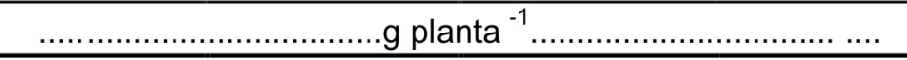 } \\
\hline \multirow{6}{*}{ Presença } & Ausência & $16,8 \mathrm{Ad}$ & $10,4 \mathrm{Ad}$ & $9,5 \mathrm{Ab}$ & $27,2 \mathrm{Ac}$ \\
\hline & Orgânica & $21,9 \mathrm{Abc}$ & $15,8 \mathrm{Abc}$ & $18,6 \mathrm{Aa}$ & $37,7 \mathrm{Ab}$ \\
\hline & Mineral NPK & $25,4 \mathrm{Aab}$ & $22,5 \mathrm{Aa}$ & $21,8 \mathrm{Aa}$ & $47,9 \mathrm{Aa}$ \\
\hline & Mineral $-\mathrm{N}$ & $23,2 \mathrm{Aa}$ & $18,9 \mathrm{Aab}$ & $22,0 \mathrm{Aa}$ & $47,1 \mathrm{Aa}$ \\
\hline & Mineral $-\mathrm{P}$ & $18,0 \mathrm{Acd}$ & 11,9 Acd & $10,4 \mathrm{Ab}$ & $29,9 \mathrm{Ac}$ \\
\hline & Mineral $-\mathrm{K}$ & $21,9 \mathrm{Abc}$ & $17,1 \mathrm{Ab}$ & $19,2 \mathrm{Aa}$ & $39,0 \mathrm{Ab}$ \\
\hline \multirow{6}{*}{ Ausência } & Ausência & $13,4 \mathrm{Abc}$ & 7,6 Abc & 9,0 Acd & $21,0 \mathrm{Ac}$ \\
\hline & Orgânica & $21,0 \mathrm{Aa}$ & $15,2 \mathrm{Aa}$ & $14,0 \mathrm{Bab}$ & $36,2 \mathrm{Aa}$ \\
\hline & Mineral NPK & $17,0 \mathrm{Bab}$ & $11,0 \mathrm{Bab}$ & $11,4 \mathrm{Bbc}$ & $28,0 \mathrm{Bb}$ \\
\hline & Mineral -N & $17,8 \mathrm{Ba}$ & $14,6 \mathrm{Ba}$ & $17,1 \mathrm{Ba}$ & $32,4 \mathrm{Bab}$ \\
\hline & Mineral -P & $6,7 \mathrm{Bd}$ & $2,5 \mathrm{Bd}$ & $3,4 \mathrm{Be}$ & $9,2 \mathrm{Bd}$ \\
\hline & Mineral -K & $12,3 \mathrm{Bc}$ & 6,3 Bcd & $6,5 \mathrm{Bde}$ & $18,6 \mathrm{Bc}$ \\
\hline
\end{tabular}

${ }^{1}$ Médias seguidas de letra maiúscula igual para o mesmo tipo de adubação na presença e ausência de calagem e letras minúsculas entre adubações dentro do mesmo nível de calagem, não diferem estatisticamente entre si pelo teste de Duncan ao nível de 5 \% de probabilidade. 
TABELA5. Volume radicular (VR), área foliar (AF) e altura (H) para mudas de Maytenus ilicifolia, cultivadas por 314 dias. Curitiba, Paraná1.

\begin{tabular}{|c|c|c|c|c|}
\hline \multicolumn{2}{|c|}{ Tratamentos } & VR & $A F$ & $\mathrm{H}$ \\
\hline Calagem & Adubação & $\mathrm{cm}^{3}$ planta $^{-1}$ & $\mathrm{~cm}^{2}$ planta $^{-1}$ & $\mathrm{~cm}_{\text {planta }}{ }^{-1}$ \\
\hline \multirow{6}{*}{ Presença } & Ausência & $21,5 \mathrm{Bd}$ & $11,9 \mathrm{AC}$ & $54,4 \mathrm{AC}$ \\
\hline & Orgânica & $40,5 \mathrm{Abc}$ & 13,8 Aab & $64,3 \mathrm{Abc}$ \\
\hline & Mineral NPK & $47,0 \mathrm{Aab}$ & $14,5 \mathrm{Aab}$ & $78,2 \mathrm{Aa}$ \\
\hline & Mineral -N & 54,2 Аа & 17,8 Аа & 76,0 Aа \\
\hline & Mineral -P & $32,2 \mathrm{Acd}$ & $12,0 \mathrm{Ac}$ & $62,0 \mathrm{Abc}$ \\
\hline & Mineral $-K$ & 48,7 Aab & $13,0 \mathrm{Abc}$ & 73,2 Aab \\
\hline \multirow{6}{*}{ Ausência } & Ausência & $33,0 \mathrm{Ab}$ & $10,1 \mathrm{Bcd}$ & $51,4 \mathrm{Ab}$ \\
\hline & Orgânica & 43,0 Aab & 12,9 Aa & $72,6 \mathrm{Aa}$ \\
\hline & Mineral NPK & $36,2 \mathrm{Ab}$ & $11,3 \mathrm{Bbc}$ & $62,5 \mathrm{Bab}$ \\
\hline & Mineral $-\mathrm{N}$ & $51,2 \mathrm{Aa}$ & 11,9 Bab & $68,5 \mathrm{Aa}$ \\
\hline & Mineral $-P$ & $9,5 \mathrm{Bc}$ & $7,00 \mathrm{Be}$ & $33,4 \mathrm{Bc}$ \\
\hline & Mineral -K & $14,7 \mathrm{Bc}$ & $9,10 \mathrm{Bd}$ & $55,8 \mathrm{Bb}$ \\
\hline
\end{tabular}

${ }^{1}$ Médias seguidas de letra maiúscula igual para o mesmo tipo de adubação na presença e ausência de calagem e letras minúsculas entre adubações dentro do mesmo nível de calagem, não diferem estatisticamente entre si pelo teste de Duncan ao nível de $5 \%$ de probabilidade.

TABELA6. Diâmetro (D), relação raiz parte aérea (R/PA) e relação altura diâmetro (H/D) para mudas de Maytenus ilicifolia, cultivadas por 314 dias. Curitiba, Paraná1.

\begin{tabular}{ccccc}
\hline \multicolumn{2}{c}{ Tratamentos } & $\mathrm{D}$ & $\mathrm{R} / \mathrm{PA}$ & $\mathrm{H} / \mathrm{D}$ \\
\hline Calagem & Adubação & $\mathrm{mm} \mathrm{planta}^{-1}$ & g planta $^{-1}$ & $\mathrm{~mm} \mathrm{~cm}^{-1}$ \\
\hline \multirow{4}{*}{ Presença } & Ausência & $5,9 \mathrm{Aa}$ & $0,35 \mathrm{Ab}$ & $9,20 \mathrm{Ab}$ \\
& Orgânica & $5,9 \mathrm{Aa}$ & $0,49 \mathrm{Aa}$ & $10,94 \mathrm{Aab}$ \\
& Mineral NPK & $6,4 \mathrm{Aa}$ & $0,46 \mathrm{Aa}$ & $12,26 \mathrm{Aa}$ \\
& Mineral $-\mathrm{N}$ & $6,3 \mathrm{Aa}$ & $0,46 \mathrm{Aa}$ & $12,25 \mathrm{Aa}$ \\
& Mineral -P & $5,2 \mathrm{Ab}$ & $0,35 \mathrm{Ab}$ & $11,92 \mathrm{Aa}$ \\
& Mineral -K & $6,4 \mathrm{Aa}$ & $0,50 \mathrm{Aa}$ & $11,46 \mathrm{Aa}$ \\
\hline \multirow{4}{*}{ Ausência } & Ausência & $5,0 \mathrm{Bcd}$ & $0,43 \mathrm{Aab}$ & $10,30 \mathrm{Ab}$ \\
& Orgânica & $5,6 \mathrm{Aab}$ & $0,38 \mathrm{Bb}$ & $12,93 \mathrm{Aa}$ \\
& Mineral NPK & $5,1 \mathrm{Bbc}$ & $0,41 \mathrm{Ab}$ & $12,18 \mathrm{Aab}$ \\
& Mineral -N & $6,0 \mathrm{Aa}$ & $0,53 \mathrm{Aa}$ & $11,56 \mathrm{Aab}$ \\
& Mineral -P & $3,2 \mathrm{Be}$ & $0,37 \mathrm{Ab}$ & $10,47 \mathrm{Ab}$ \\
& Mineral -K & $4,5 \mathrm{Bd}$ & $0,35 \mathrm{Bb}$ & $12,34 \mathrm{Ab}$ \\
\hline
\end{tabular}

${ }^{1}$ Médias seguidas de letra maiúscula igual para o mesmo tipo de adubação na presença e ausência de calagem e letras minúsculas entre adubações dentro do mesmo nível de calagem, não diferem estatisticamente entre si pelo teste de Duncan ao nível de $5 \%$ de probabilidade.

essa espécie quando cultivada em solo adubado necessita de uma correção adequada em $P$ mantendo o equilíbrio entre os nutrientes. Tucci (1991) comenta que após a correção da acidez, o fator nutricional que primeiro limita o crescimento de plantas é o baixo teor de fósforo disponível nos solos, como verificado no solo desse estudo. Estes resultados podem ser indicativos da importância do manejo adequado da adubação fosfatada, para estas condições, devido ao importante papel do $\mathrm{P}$ no crescimento das plantas (Marschner, 1995).

Dentre os vários sintomas de deficiência de $\mathrm{N}$, pode-se destacar a diminuição no crescimento, como observado por Venturin et al. (1999) em mudas de angico amarelo (Peltophorum dubium) e Nicoloso et al. (1999) em plantas jovens de grápia (Apuleia leiocarpa). No entanto, nesse trabalho, as plantas submetidas ao tratamento com omissão de $\mathrm{N}$ não 
demonstraram estes sintomas, uma vez que todas as variáveis (Tabela 4, 5 e 6) foram superiores ou iguais em relação a adubação mineral completa. Este comportamento pode ser explicado pela possibilidade de um adequado suprimento desse nutriente pela matéria orgânica (Tabela 3) desse solo. Provavelmente esta foi a causa da resposta diferenciada da omissão de N nesse estudo em relação aos trabalhos de Pintro et al. (1998) em mudas de erva-mate (Ilex paraguariensis), Souza et al. (2006) em ipê-roxo (Tabebuia impetiginosa), Venturin et al. (1999) em angico-amarelo (Peltophorum dubium) e Braga et al. (1995) em acácia (Acacia mangium), quaresmeira (Tibouchina granulosa) e pereira (Platycyamus regnellii), visto que os substratos utilizados nesses trabalhos possuíam teor de matéria orgânica considerado baixo a médio.

A omissão de $\mathrm{K}$ na presença da calagem afetou negativamente a MSC e MSPA(Tabela 4), não diferindo da mineral completa para as demais variáveis. Renó et al. (1997) não obtiveram redução do crescimento em H, MSPA, MSR e R/PA para canafístula (Senna multijuga), cedro (Cedrela fissillis), pau-ferro (Caesalpinea ferrea) e jacaré (Piptadenea gonoacantha), quando omitiram $\mathrm{K}$ da adubação, justificando esta resposta pela presença de mica no solo, a qual teria suprido satisfatoriamente estas espécies em K. Dessa forma, em trabalhos futuros faz-se necessário o monitoramento a fim de verificar a necessidade de adubação de reposição, visto que as reservas de $\mathrm{K}$ do solo tendem a diminuir. Já na ausência da calagem, a omissão de K prejudicou a maioria das variáveis de crescimento, com exceção da $\mathrm{H}, \mathrm{R} / \mathrm{PA}$ e $\mathrm{H} / \mathrm{D}$, as quais não diferiram da adubação mineral completa (Tabelas 4, 5 e 6).

Estes resultados podem ser reflexos do crescimento radicular das plantas, onde na presença da calagem observa-se igualdade entre a adubação mineral completa e omissão de K para a MSR e VR. Já na ausência da calagem, as variáveis de crescimento relacionadas às raízes, foram superiores na adubação mineral completa em relação à omissão de K (Tabelas 4 e 5), o que provavelmente, pode ter sido a causa do efeito negativo observado para a maioria das outras variáveis de crescimento analisadas nessa condição de omissão de K sem calagem.

\section{CONCLUSÃO}

A calagem quando utilizada como prática isolada trouxe poucos benefícios significativos ao crescimento das plantas. No entanto, quando associada à adubação esta favorece tanto a mineral quanto a orgânica.

Em condições de adubação mineral, independente da presença ou não da calagem, o fósforo mostra-se importante na nutrição de plantas jovens de espinheira-santa.

\section{AGRADECIMENTO}

À empresa Baldo S.A. Indústria Comércio e Exportação, filial de São Mateus do Sul-PR, pela coleta das sementes e produção das mudas e apoio durante todo o desenvolvimento desse estudo.

\section{REFERÊNCIA}

BITTENCOURT, J.V.M. Variabilidade genética em populações de Maytenus ilicifolia por meio de marcadores RADP. 2000. 58p. Dissertação (Mestrado em Agronomia) - Setor de Ciências Agrárias, Universidade Federal do Paraná, Curitiba.

BLANK, A.F. et al. Efeitos da adubação química e da calagem na nutrição de melissa e hortelã-pimenta. Horticultura Brasileira, v.24, n.2, p.195-8, 2006.

BRAGA, F.A. et al. Exigências nutricionais de quatro espécies florestais. Revista Árvore, v.19, n.1, p.18-31, 1995.

CARLINI, E.L.A. (Coord.). Estudo de ação antiúlcera gástrica de plantas brasileiras: Maytenus ilicifolia (Espinheira-Santa) e outras. Brasília: CEME/AFIP. 1988. 87p.

CARNEIRO, J.G.A. Produção e controle de qualidade de mudas florestais. Curitiba: UFPR/FUPEF, Campos: UENF, 1995. 451p.

CARVALHO, P.E.R. Levantamento florístico da região de Irati-PR. Curitiba: EMBRAPA/CNPF. 1980. 44p. (Circular Técnica, 03).

CARVALHO-OKANO, R.M. Estudos taxonômicos do gênero Maytenus Mol. Emend. Mol. (Celastraceae) do Brasil extra-amazônico. 1992. 257p. Tese (Doutorado em Ciências - Biologia Vegetal) - Instituto de Biologia da Universidade Estadual de Campinas, Campinas. COMISSÃO DE QUÍMICA E FERTILIDADE DO SOLO CQFSRS/SC. Manual de adubação e calagem para os Estados do Rio Grande do Sul e Santa Catarina. 10.ed. Porto Alegre: Sociedade Brasileira de Ciência do Solo Núcleo Regional Sul, 2004. 394p.

CORRÊA JÚNIOR, C.; MING, L.C.; SCHEFFER, M.C. Cultivo de plantas medicinais, condimentares e aromáticas. Curitiba: EMATER-PR, 1991.151p.

CRUZ, C.A.F. et al. Efeito de diferentes níveis de saturação por bases no desenvolvimento e qualidade de mudas de ipê-roxo (Tabebuia impetiginosa (Mart.) Standley). Scientia Forestalis, n.66, p.100-7, 2004.

EMBRAPA. Manual de métodos de análise de solo.2.ed. Rio de Janeiro: EMBRAPA, 1997.212p.

EMBRAPA. Núcleo Tecnológico para Informática. Campinas: SOC - Software Científico, 1990.

ERNANI, P.R.; GIANELLO, C. Diminuição do alumínio trocável do solo pela incorporação de esterco de bovinos e camas de aviário. Revista Brasileira de Ciência do Solo, v.7, n.2, p.161-5, 1983.

FRANCHINI, J.C. et al. Alterações químicas em solos ácidos após a aplicação de resíduos vegetais. Revista Brasileira de Ciência do Solo, v.23, n.3, p.533-42, 1999. FURTINI NETO, A.E. et al. Acidez do solo, crescimento e nutrição mineral de algumas espécies arbóreas, na fase de muda. Cerne, v.5, n.2, p.1-12, 1999.

GALVÃO, F.; KUNIYOSHI, Y.S.; RODERJAN, C.V. 
Levantamento fitossociológico das principais associações arbóreas da Floresta Nacional de Irati-PR. Floresta, v.19, n.1-2, p.30-49, 1989.

KIEHL, E.J. Fertilizantes organominerais. Piracicaba: Agronômica Ceres, 1993. 189p.

MARSCHNER, $\mathrm{H}$. Mineral nutrition of higher plants. London: Academic Press, 1995. 889p.

MELLO, S.C.; VITTI, G.C. Influência de materiais orgânicos no desenvolvimento do tomateiro e nas propriedades químicas do solo em ambiente protegido. Horticultura Brasileira, v.20, n.3, p.452-8, 2002.

MENEZES, A.J. et al. Fitotecnia de medicinais: influência da adubação na produção de fitomassa de Maytenus aquifolia. In: SIMPÓSIO DE PLANTAS MEDICINAIS DO BRASIL, 12., 1992, Curitiba. Anais... Curitiba, 1992.p.239. MIYAZAWA, M.; CHIERICE, G.O.; PAVAN, M.A. Amenização da toxicidade de alumínio às raízes do trigo pela complexação com ácidos orgânicos. Revista Brasileira de Ciência do Solo, v.16, n.2, p.209-15, 1992.

MONTANARI JÚNIOR, I. Plantas medicinais. In: SEMINÁRIO NACIONAL RECURSOS FLORESTAIS DA MATAATLÂNTICA, 1., 1999, São Paulo. Anais... São Paulo, 1999. p.50-8.

MONTANARI JÚNIOR, I.; SCHEFFER, M.C.; RADOMSKI, M.I. Cultivo de espinheira-santa. In: REIS, M.S.; SILVA, S.R. (Orgs.). Conservação e uso sustentável de plantas medicinais e aromáticas: Maytenus spp., Espinheirasanta. Brasília, 2004. p.163-80.

MOTTA. A.C.V.; SERRAT, B.M. Princípios de adubação. In:LIMA, M.R. et al. (Eds.). Diagnóstico e recomendação de manejo do solo: aspectos teóricos e metodológicos. Curitiba: UFPR/Setor de Ciências Agrárias, 2006. p.14390.

NICOLOSO, F.T. et al. Exigências nutricionais da grápia (Apuleia leiocarpa Vog. Macbride) em solo Podzólico vermelho amarelo. Ciência Rural, v.29, n.2, p.225-31, 1999.

OLIVEIRA, Y.M.M.; ROTTA, E. Área de distribuição natural da erva-mate (Ilex paraguariensis St. Hil.). In: SEMINÁRIO SOBREATUALIDADES E PERSPECTIVAS FLORESTAIS 10., 1985, Curitiba. Anais... Curitiba: Embrapa, 1985. p.1736.

PINTRO, J.C.; MATUMOTO-PINTRO, P.T.; SCHWANESTRADA, K.R.F. Crescimento e desenvolvimento de mudas de erva-mate (Ilex paraguariensis St. Hil.) cultivadas em solo sob diferentes níveis de fertilidade. Acta Scientiarum, v.20, n.3, p.285-9, 1998.

RADOMSKI, M.I. Teores foliares de silício, taninos e lignina, em Maytenus ilicifolia Martius ex Reiss (Espinheira-santa), em função de variáveis ambientais e genéticas. 2006. 116p. Tese (Doutorado em Agronomia/Horticultura) - Faculdade de Ciências Agronômicas, Universidade Estadual Paulista, Botucatu. REISSMANN, C.B. et al. Production and foliar N, P, K, Ca and $\mathrm{Mg}$ levels in erva-mate (Ilex paraguariensis St. Hil.) related to increasing base saturation levels. Arquivos de Biologia e Tecnologia, v.40, n.1, p.241-9, 1997.

RENÓ, N.B. et al. Limitações nutricionais de quatro espécies arbóreas nativas em Latossolo VermelhoAmarelo. Pesquisa Agropecuária Brasileira, v.32, n.1, p.17-25, 1997.

SANTIN, D. et al. Uso de substratos alternativos com solo no desenvolvimento inicial de mudas de erva-mate (Ilex paraguariensis St. Hill). In: SIMPÓSIO LATINOAMERICANO SOBRE MANEJO FLORESTAL, 3., 2004, Santa Maria. Anais... Santa Maria, 2004. p.200-5.

SCHEFFER, M.C. Uso tradicional e atual de espécies de Maytenus. In: REIS, M.S.; SILVA, S.R. (Orgs.). Conservação e uso sustentável de plantas medicinais e aromáticas: Maytenus spp., espinheira-santa. Brasília: Ibama, 2004. p.53-66.

SELBACH, P.A.; SÁ, E.L.S. Fertilizantes orgânicos, organo-minerais e agricultura orgânica. In: BISSANI, C.A. et al. Fertilidade dos solos e manejo da adubação de culturas. Porto Alegre: Gênesis, 2004. p.175-86.

SILVA JUNIOR, A.A. Essentia herba - plantas bioativas. Florianópolis: Epagri, 2003. 441p.

SOUSA, D.M.G.; MIRANDA, L.N.; OLIVEIRA, S.A. Acidez do solo e sua correção. In: NOVAIS, R.F. et al. (Eds.). Fertilidade do Solo. Viçosa: Sociedade Brasileira de Ciência do Solo, 2007. p.205-74.

SOUZA, P.A.; VENTURIN, N.; MACEDO, R.L.G. Adubação mineral do ipê-roxo (Tabebuia impetiginosa). Ciência Florestal, v.16, n.3, p.261-70, 2006.

STEENBOCK, W. Fundamentos para o manejo de populações naturais de espinheira-santa, Maytenus ilicifolia Mart. ex Reiss. (Celastraceae). 2003. 145p. Dissertação (Mestrado em Recursos Genéticos Vegetais) - Universidade Federal de Santa Catarina, Florianópolis.

STEENBOCK, W.; REIS, M.S. Manejo de populações naturais de espinheira-santa. In: REIS, M.S.; SILVA, S.R. (Orgs.). Conservação e uso sustentável de plantas medicinais e aromáticas: Maytenus spp., espinheirasanta. Brasília: Ibama, 2004. p.145-61.

STORCK, L. et al. Experimentação vegetal. Santa Maria: UFSM, 2000. 198p.

TEDESCO, M.J.; BISSANI, C.A. Acidez dos solos e seus efeitos nas plantas. In: BISSANI, C.A. et al. Fertilidade dos solos e manejo da adubação de culturas. Porto Alegre: Gênesis, 2004. p.75-92.

TUCCI, C.A.F. Disponibilidade de fósforo em solos da Amazônia. 1991. 142p. Tese (Doutorado em Solos Nutrição de Plantas) - Universidade Federal de Viçosa, Viçosa.

VENTURIN, N. et al. Adubação mineral do angicoamarelo (Peltophorum dubium (Spreng.) Taub.) Pesquisa Agropecuária Brasileira, v.34, n.3, p.441-8, 1999. 